\title{
Gene expression profiling analysis of lung adenocarcinoma
}

\author{
H. $\mathrm{Xu}^{1,2}$, J. $\mathrm{Ma}^{1}$, J. $\mathrm{Wu}^{1}$, L. Chen ${ }^{1}$, F. Sun ${ }^{1}$, C. $\mathrm{Qu}{ }^{1}$, D. Zheng ${ }^{1}$ and S. $\mathrm{Xu}^{1}$ \\ ${ }^{1}$ Department of Thoracic Surgery, Harbin Medical University Cancer Hospital, Harbin, Heilongjiang, China \\ ${ }^{2}$ Laboratory of Medical Genetics, Harbin Medical University, Harbin, Heilongjiang, China
}

\begin{abstract}
The present study screened potential genes related to lung adenocarcinoma, with the aim of further understanding disease pathogenesis. The GSE2514 dataset including 20 lung adenocarcinoma and 19 adjacent normal tissue samples from 10 patients with lung adenocarcinoma aged 45-73 years was downloaded from Gene Expression Omnibus. Differentially expressed genes (DEGs) between the two groups were screened using the $t$-test. Potential gene functions were predicted using functional and pathway enrichment analysis, and protein-protein interaction (PPI) networks obtained from the STRING database were constructed with Cytoscape. Module analysis of PPI networks was performed through MCODE in Cytoscape. In total, 535 upregulated and 465 downregulated DEGs were identified. These included ATP5D, UQCRC2, UQCR11 and genes encoding nicotinamide adenine dinucleotide $(\mathrm{NADH})$, which are mainly associated with mitochondrial ATP synthesis coupled electron transport, and which were enriched in the oxidative phosphorylation pathway. Other DEGs were associated with DNA replication (PRIM1, MCM3, and RNASEH2A), cell surface receptor-linked signal transduction and the enzyme-linked receptor protein signaling pathway (MAPK1, STAT3, RAF1, and JAK1), and regulation of the cytoskeleton and phosphatidylinositol signaling system (PIP5K1B, PIP5K1C, and PIP4K2B). Our findings suggest that DEGs encoding subunits of NADH, PRIM1, MCM3, MAPK1, STAT3, RAF1, and JAK1 might be associated with the development of lung adenocarcinoma.
\end{abstract}

Key words: Lung adenocarcinoma; Pathogenesis; Differentially expressed genes; Protein-protein interaction; Network module

\section{Introduction}

Lung cancer is the leading cause of cancer deaths among men and women worldwide. The incidence of lung cancer has shown a rising trend in China, with an average annual growth of $1.63 \%$ (1). Pathologically, lung cancer can be divided into small cell and the more common non-small cell histological types. The survival prognosis of non-small cell lung cancer (NSCLC) patients is extremely poor, with an average annual 5-year survival rate of less than $15 \%$ (2).

The development of lung adenocarcinoma is a multifactor and multistage process, with genetic instability considered to be the key cause. In recent years, important progress has been made in understanding the molecular mechanism of lung adenocarcinoma. Kris et al. (3) reported that $60 \%(252 / 422)$ of lung adenocarcinoma tissues harbor a driver mutation, including those in genes encoding Kirsten rat sarcoma viral oncogene (KRAS; $25 \%$ ), epidermal growth factor receptor (EGFR; 23\%), anaplastic lymphoma kinase $(A L K ; 6 \%)$ and proto-oncogene B-Raf (BRAF; 3\%). Among these, the EGFR pathway is the main signaling pathway of lung cancer, and the mutation rate of its genes reaches $70 \%-80 \%$ (4). EGFR mutations are usually heterozygotic because the mutant allele is also coupled with gene amplification. The kinase activity increase of EGFR can lead to the hyperactivation of downstream signal pathways that enhance cell survival. KRAS mutations account for $30 \%-35 \%$ of lung adenocarcinoma genetic variation (5). Around $97 \%$ of KRAS mutations in NSCLC occur in codons 12 or 13 (6), and Mills et al. (7) have shown that the sensitive detection of KRAS codon 12 mutations in bronchoalveolar lavage can help diagnose lung cancer. $A L K$ fusions are observed in $\sim 4 \%$ of NSCLC patients (8), resulting from an inversion of EML4 and $A L K$ gene on the short arm of chromosome 2 which constitutively activates the kinase and protein oligomerization (9).

As well as the above genes and pathways, other molecular changes can bring about lung adenocarcinoma, such as mutations in ROS (10,11), ERCC1 (12), RB (13), AKT (14), PTEN (15), and MAP2K1 (16). Stearman et al. (17) compared orthologous gene expression between human pulmonary adenocarcinoma and a urethane-induced murine model, and identified 409 gene classifiers that showed significant $(P<0.0001)$ and positive correlation in expression between the two species. Moreover, the detection of prostacyclin synthase was found to have a significant prognostic

Correspondence: S. Xu: <shidongxuxux@163.com>

Received April 15, 2015 | Accepted August 5, 2015 
value in patient survival. However, no further investigations have been carried out into changes in metabolic pathways or genes involved in human lung adenocarcinoma.

In this study, therefore, we aimed to obtain an improved insight into lung adenocarcinoma by searching microarray data for differentially expressed genes (DEGs) between lung adenocarcinoma and adjacent normal tissue samples. We also constructed a protein-protein interaction (PPI) network, and performed functional and pathway enrichment analyses of network modules.

\section{Material and Methods}

\section{Affymetrix microarray data}

The expression profile data of GSE2514 were obtained from a public functional genomics data repository Gene Expression Omnibus (GEO) database (http://www.ncbi.nlm. nih.gov/geo/) (17), which was based on the platform of the Affymetrix Human Genome U95 Version 2 Array. A total of 39 human tissue samples were available for further analysis, of which 20 were lung adenocarcinoma samples and 19 were adjacent normal tissue samples from five males and five females with lung adenocarcinoma, aged 45-73 years. All patients participating in this study were enrolled in a protocol approved by the local Colorado Multiple Institutional Review Board for the use of remnant tissues with anonymization and analysis of specimens and clinical data. Tumors were histologically classified according to World Health Organization guidelines and staged according to the tumor-node-metastasis classification. With the exception of two stage III tumors, most tumors were low stage and low to intermediate grade.

Affymetrix CEL files and probe annotation files were downloaded, and gene expression data of all samples were preprocessed using the GeneChip Robust Multi Array algorithm in the Affy software package (18).

\section{DEG screening}

The $t$-test was used to identify genes that were significantly differentially expressed between lung tumor samples and adjacent normal tissue samples. The raw P-value was adjusted by the Benjamin and Hochberg method (19), and only genes following the cut-off criteria of $\left[\log _{2} \mathrm{FC}\right.$ (fold change) $>0.5$ and adjusted $\mathrm{P}<0.05$ were selected as DEGs.

\section{Gene ontology (GO) and pathway enrichment analyses}

The Database for Annotation, Visualization and Integrated Discovery (DAVID) gene functional classification database now provides a set of comprehensive functional annotation tools for investigators to comprehend the biological meanings behind many genes. Kyoto Encyclopedia of Genes and Genomes (KEGG) pathway enrichment analysis (20) was conducted to identify significant pathways for DEGs. A $\mathrm{P}<0.05$ was used as the cut-off criterion for $\mathrm{GO}$ and KEGG pathway enrichment analyses using default parameters by DAVID.

\section{PPI network construction}

The Search Tool for the Retrieval of Interacting Genes (STRING) database provides both experimental and predicted interaction information. This database was used to analyze PPIs for DEGs by calculating their Required Confidence score; a score $>0.4$ was chosen as the cut-off criterion. PPI networks of upregulated and downregulated DEGs were then respectively visualized by Cytoscape (http://cytoscape.org/), which is an open source software for visualizing complex networks and integrating them with any type of attribute data. Hub proteins (essential high-degree proteins in PPI networks) $(21,22)$ were found by counting the connectivity degree of each network node based on the scale-free property of interaction networks. The connectivity degree of each node represents the number of interactions the node has with other nodes.

\section{Screening and analysis of network modules}

Network modules were obtained based on the MCODE analysis of original PPI networks. Default parameters (Degree Cutoff: 2, Node Score Cutoff: 0.2, K-Core: 2, Max. Depth: 100) were used as the cut-off criteria for network module screening.

To obtain a better understanding at the molecular level of gene function and to identify pathways closely associated with DEGs, the functional annotation and pathway enrichment analysis of network modules with higher MCODE scores were performed using online DAVID software with a threshold of $\mathrm{P}<0.05$.

\section{Results}

\section{DEGs between lung tumor and healthy lung tissue cells}

After data preprocessing, 11,551 probes were obtained. Based on the cut-off criteria, 1000 DEGs including 535 that were upregulated and 465 downregulated were screened.

\section{GO and KEGG pathway enrichment analyses of upregulated and downregulated DEGs}

GO terms of upregulated DEGs were significantly related to RNA processing ( $P=3.89 E-09)$, RNA splicing $(\mathrm{P}=3.27 \mathrm{E}-07)$, oxidative phosphorylation $(\mathrm{P}=3.51 \mathrm{E}-07)$, and the electron transport chain $(\mathrm{P}=6.76 \mathrm{E}-07$; Supplementary Table S1). GO terms of downregulated DEGs were mainly related to the regulation of cell motion $(P=7.21 \mathrm{E}-05)$, morphogenesis of a branching structure $(P=8.02 E-05)$, and lung development ( $P=7.37 E-04$; Supplementary Table S2).

Upregulated DEGs were enriched in 11 pathways, and most significantly in oxidative phosphorylation ( $P=4.67 E-07)$, Parkinson's disease ( $P=5.63 E-07)$, and Huntington's disease $(P=1.11 E-05$; Figure $1 A)$, while downregulated $D E G s$ 

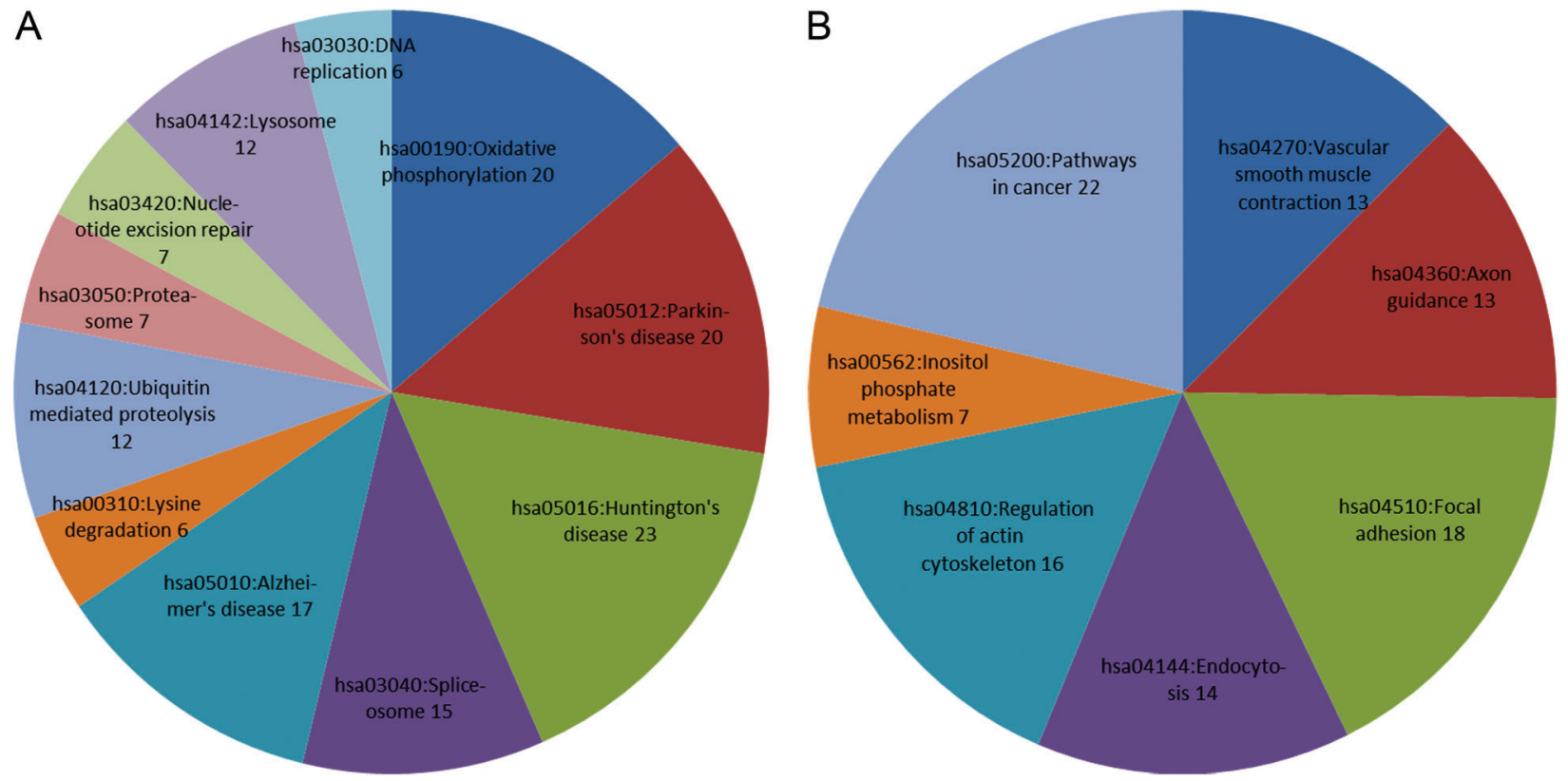

Figure 1. KEGG pathway enrichment analysis for upregulated differentially expressed genes $(A)$ and downregulated differentially expressed genes $(B)$.

were enriched in seven pathways, most significantly in vascular smooth muscle contraction ( $P=1.29 E-03)$, axon guidance $(P=3.80 E-03)$, and focal adhesion $(P=6.74 E-03$; Figure 1B).

\section{Construction and analysis of PPI networks}

PPI networks for upregulated and downregulated DEGs consisted of 1,591 and 661 pairs of PPIs, respectively (Figure 2A and B).

The connectivity degree of certain genes exceeded 20 , including DHX15, NDUFS3, PSMC6, UBE2C, EIF4G1, $D H X 9, P S M C 3$, and HNRNPA2B1 in the upregulated PPI network, and MAPK1, IL6, FN1, MAPK14, STAT3, and VWF in the downregulated PPI network (Table 1).

\section{Analysis of network modules}

A total of 24 modules including 14 upregulated and 10 downregulated network modules were obtained using default criteria. Among these, five upregulated modules (up-1, up-2, up-3, up-4, and up-5) with nodes $>5$ and a MCODE score $>6$ (Figure 3 ), and six downregulated modules ( $d-1, d-2, d-3, d-4, d-5$, and $d-6)$ with nodes $>3$ and a MCODE score $>3$ (Figure 4) were selected for enrichment analysis.

Functional enrichment analysis for two upregulated modules (up-1 and up-2) with a higher enrichment score showed that the genes in module up-1 (e.g., $D H X 9$, HNRNPA2B1, HNRNPR, GTF2F2, and SNRNP40) were mainly enriched in RNA splicing $(P=1.53 E-17)$ and $m R N A$ processing $(\mathrm{P}=3.94 \mathrm{E}-14)$ pathways. Genes in module up-2 (ATP5D, UQCRC2, NDUFS7, NDUFA2, UQCR11, NDUFB8, NDUFV1, NDUFV2, NDUFA7, NDUFS3, NDUFA1, and NDUFB1) were mainly related to mitochondrial ATP synthesis coupled electron transport $(P=1.12 E-14)$, the electron transport chain $(P=7.35 \mathrm{E}-14)$, and mitochondrial electron transport $(P=9.94 \mathrm{E}-14$; Table 2$)$. There were no significant GO terms for genes in modules up-3 or up-4; moreover, the enrichment score of module up -5 was much lower than that of modules up-1 and up-2, so module up-5 GO terms are not listed in Table 2.

Because of their higher enrichment scores and gene numbers, modules up-1 and up-2 were selected for further pathway enrichment analysis. DEGs in module up-1, such as POLR2G, POLR2F, DDX23, PRIM1, MCM3, TK1, $S N R N P 40, S N R N P 70$, and RNASEH2A, were significantly enriched in three pathways of pyrimidine metabolism $(P=1.15 E-03)$, DNA replication $(P=5.24 E-03)$, and spliceosome ( $\mathrm{P}=4.41 \mathrm{E}-02)$. DEGs in module up-2, such as ATP5D, UQCRC2, NDUFA2, NDUFB8, NDUFA7, COX4I1, NDUFA1, NDUFB1, NDUFS7, UQCR11, NDUFV1, NDUFV2, and NDUFS3, were enriched in the pathways of oxidative phosphorylation ( $\mathrm{P}=3.31 \mathrm{E}-13)$, Parkinson's disease $(P=3.81 E-13)$, Alzheimer's disease $(P=3.25 E-11)$, and Huntington's disease ( $P=7.35 \mathrm{E}-11$; Table 3$)$.

The enriched functions for genes in two downregulated modules ( $d-1$ and $d-5)$ with higher enrichment scores showed that genes in module d-1 (e.g., MAPK1, MAPK14, MITF, RAF1, JAK1, HBEGF, ROS1, and STAT3) were 

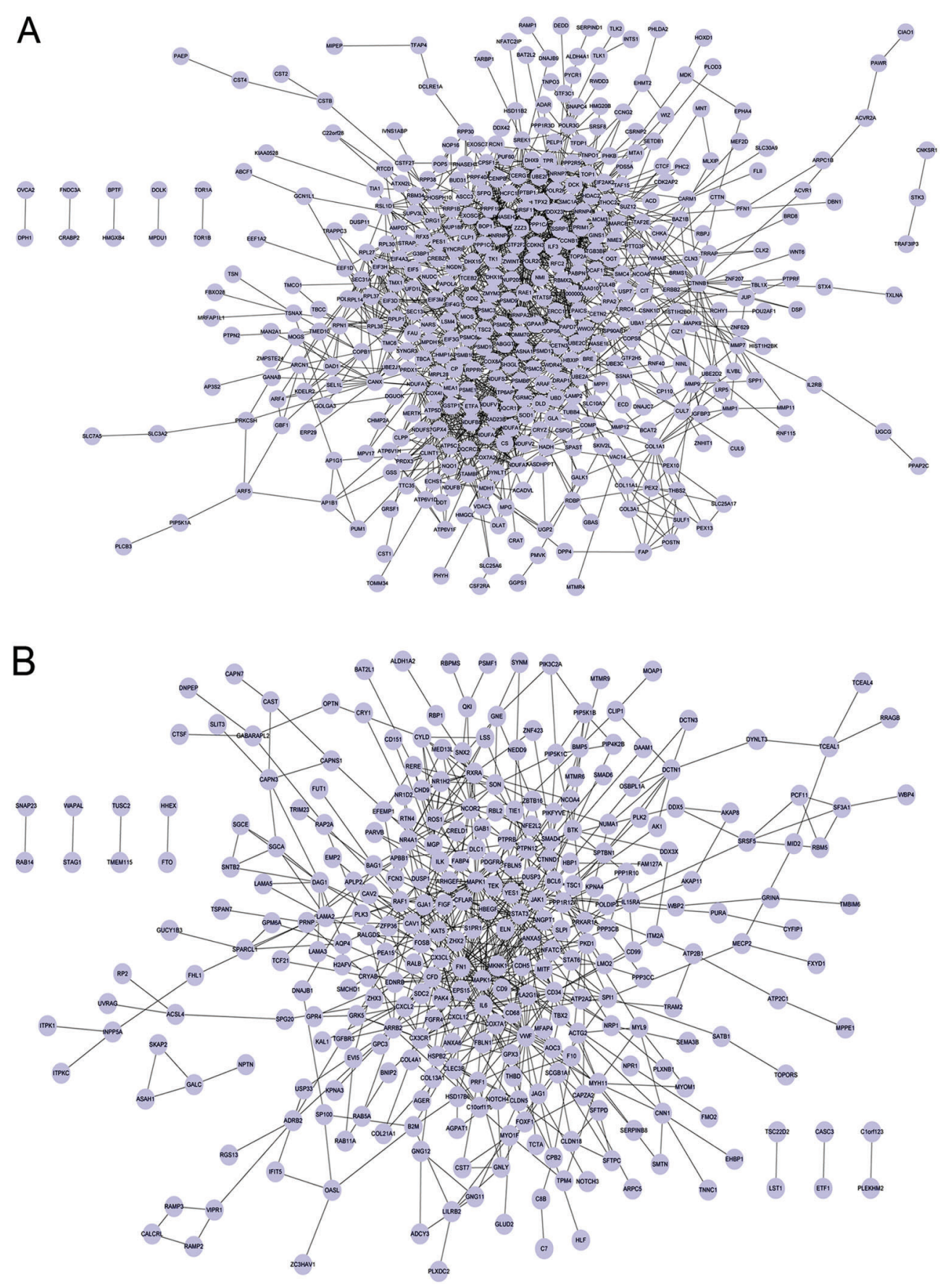

Figure 2. Protein-protein interaction network for upregulated differentially expressed genes $(A)$ and downregulated differentially expressed genes $(B)$.

related to cell surface receptor linked signal transduction $(P=1.71 E-03)$, and enzyme-linked receptor protein signaling pathway $(P=2.02 \mathrm{E}-03)$; while genes in module $\mathrm{d}-5$ (e.g., PIP5K1B, PIP5K1C, PIP4K2B, CXCL12, and FN1) were mainly enriched in phosphatidylinositol metabolic processes $(\mathrm{P}=4.24 \mathrm{E}-04)$, glycerolipid metabolic processes
( $P=1.88 E-03)$, and cell morphogenesis ( $P=2.06 E-02$; Table 4). There were no significant $\mathrm{GO}$ terms for genes in modules $\mathrm{d}-2$, d-3, d-4, or d-6.

Similarly, because of their higher enrichment scores and gene numbers, modules $d-1$ and $d-5$ were selected for further pathway enrichment analysis. Five pathways 
Table 1. Differentially expressed genes with the top $10 \%$ connectivity degree in upregulated and downregulated protein-protein interaction networks.

\begin{tabular}{|c|c|c|c|c|c|c|c|c|}
\hline Network & ID & Degree & ID & Degree & ID & Degree & ID & Degree \\
\hline \multirow[t]{11}{*}{ Upregulated } & $D H \times 15$ & 28 & SRSF11 & 20 & NDUFB8 & 18 & RPL30 & 16 \\
\hline & NDUFS3 & 26 & GTF2F2 & 20 & PAICS & 18 & PTBP1 & 16 \\
\hline & PSMC6 & 25 & CPSF1 & 20 & UQCR11 & 18 & MCM3 & 16 \\
\hline & UBE2C & 25 & NDUFV1 & 19 & PRIM1 & 18 & ZWINT & 16 \\
\hline & EIF4G1 & 24 & SNRNP70 & 19 & HNRNPR & 18 & ATP5D & 16 \\
\hline & $D H X 9$ & 23 & PSMD10 & 19 & EIF3G & 17 & SNRNP40 & 16 \\
\hline & PSMC3 & 23 & SMC1A & 19 & NDUFV2 & 17 & PABPN1 & 16 \\
\hline & HNRNPA2B1 & 22 & CCNB1 & 19 & COX4I1 & 17 & TOP1 & 16 \\
\hline & POLR2G & 21 & UQCRC2 & 18 & EIF4A3 & 17 & RNASEH2A & 16 \\
\hline & TOP2A & 21 & POLR2F & 18 & NDUFA1 & 17 & & \\
\hline & PSMB6 & 21 & $D D \times 23$ & 18 & $F A U$ & 16 & & \\
\hline \multirow[t]{6}{*}{ Downregulated } & MAPK1 & 41 & $C D 34$ & 19 & $R A F 1$ & 13 & $E L N$ & 12 \\
\hline & IL6 & 33 & HBEGF & 16 & BCL6 & 13 & ANGPT1 & 11 \\
\hline & FN1 & 32 & CAV1 & 16 & $A R R B 2$ & 13 & $M G P$ & 11 \\
\hline & MAPK14 & 25 & $Z H X 2$ & 15 & $R X R A$ & 12 & $S M A D 4$ & 11 \\
\hline & STAT3 & 22 & CXCL12 & 14 & $J A K 1$ & 12 & $N R 1 H 2$ & 11 \\
\hline & $V W F$ & 20 & NCOR2 & 14 & $\mathrm{CDH} 5$ & 12 & SDC2 & 11 \\
\hline
\end{tabular}
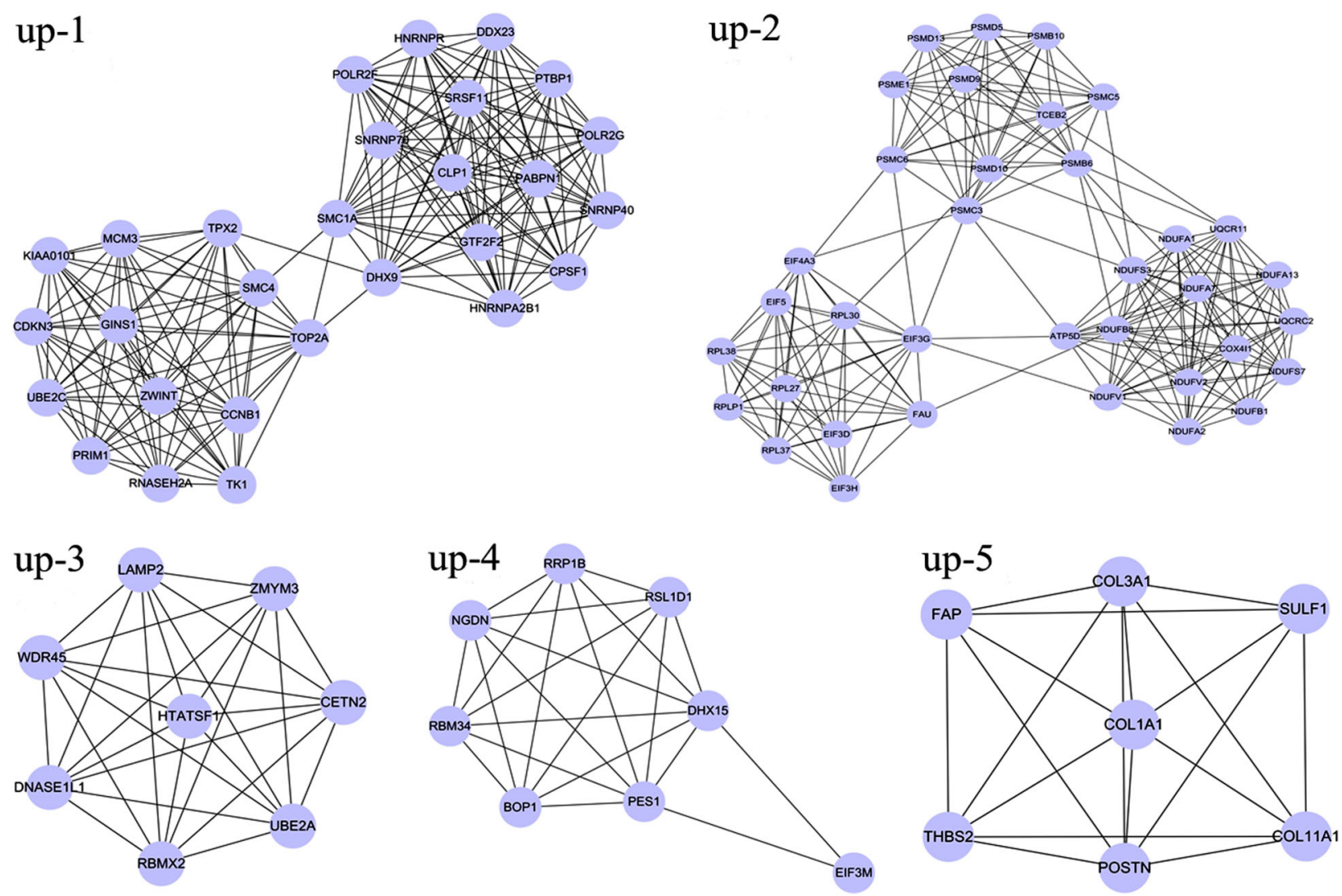

Figure 3. Modules of the protein-protein interaction network for upregulated differentially expressed genes. 
d-1

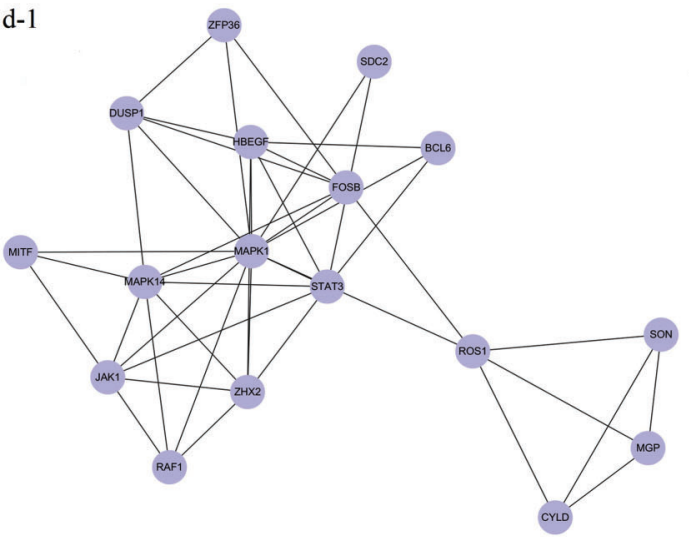

d-2

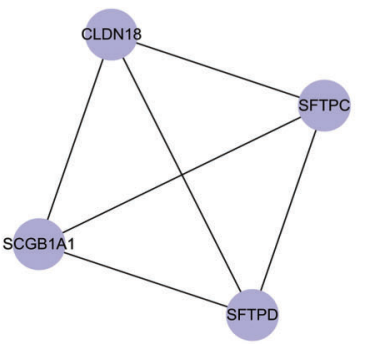

d-3

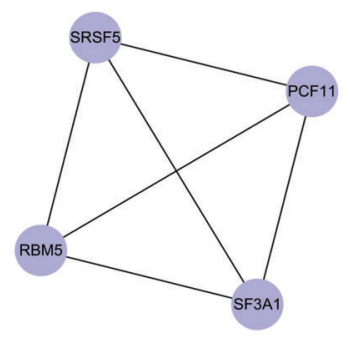

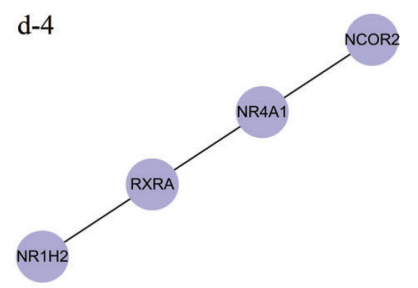

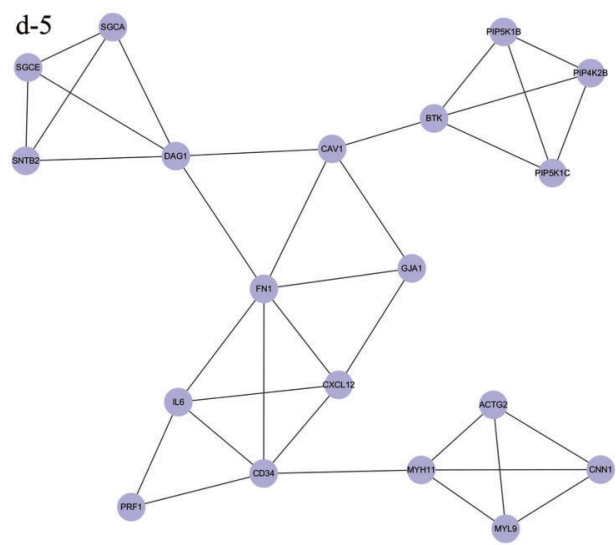

d-6

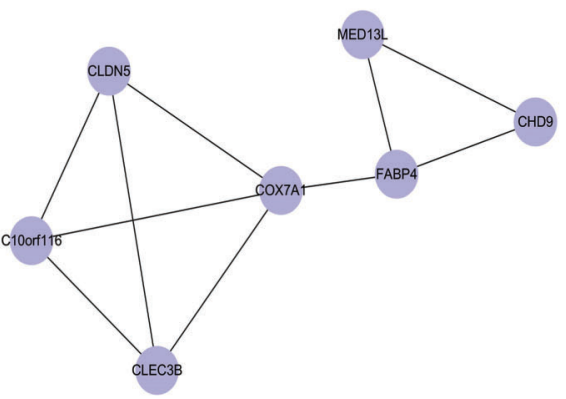

Figure 4. Modules of the protein-protein interaction network for downregulated differentially expressed genes.

were enriched for genes in the module d-1 (MAPK1, MITF, $R A F 1, J A K 1$, and STAT3): pancreatic cancer ( $P=5.47 \mathrm{E}-04)$, melanoma $(P=8.52 \mathrm{E}-03)$, melanogenesis $(P=1.48 \mathrm{E}-02)$, acute myeloid leukemia ( $P=7.67 \mathrm{E}-03)$, and cancer pathways $(P=3.69 E-03)$. Four pathways were enriched for genes in module d-5 (e.g., PIP5K1B, PIP5K1C, FN1, and PIP4K2B): regulation of the actin cytoskeleton $(P=4.61 E-03)$, inositol phosphate metabolism ( $P=1.56 E-02)$, the phosphatidylinositol signaling system ( $P=2.90 \mathrm{E}-02)$, and Fc gamma R-mediated phagocytosis ( $\mathrm{P}=4.57 \mathrm{E}-02$; Table 5$)$.

\section{Discussion}

In this study, 535 genes were identified as significantly upregulated and 465 as downregulated in lung adenocarcinoma samples compared with normal adjacent tissue samples. Based on functional and pathway enrichment analyses of two upregulated modules, the identified DEGs (ATP5D, UQCRC2, NDUFA2, NDUFB8, NDUFA7, NDUFA1, NDUFB1, NDUFS7, UQCR11, NDUFV1, NDUFV2, and NDUFS3) were mainly related to mitochondrial ATP synthesis coupled electron transport, the respiratory electron transport chain, and mitochondrial electron transport. These genes were therefore enriched in the oxidative phosphorylation pathway.

DEGs such as NDUFA2, NDUFB8, NDUFA7, NDUFA1, NDUFB1, NDUFS7, NDUFV1, NDUFV2, and NDUFS3 jointly encode subunits of nicotinamide adenine dinucleotide (NADH):ubiquinone oxidoreductase (complex I) (23). NADH is the entry enzyme of mitochondrial oxidative phosphorylation (24), which plays a key role in mitochondrial respiration. Mitochondrial respiration is thought to be vital to the bioenergetics of cancer cells, with breast, glioma, and cervical cancer cells shown to be highly reliant on mitochondrial respiration for ATP generation (25-27). It was also shown that mitochondrial respiration is substantially enhanced in NSCLC cells (28), while inhibition of mitochondrial electron transport prevents the growth of human lung cancer A549 cells (29). NDUFS1 is already considered to be a prognostic marker for NSCLC (30), so we predict that the DEGs NDUFA2, NDUFB8, NDUFA7, NDUFA1, NDUFB1, NDUFS7, NDUFV1, NDUFV2, and NDUFS3 play an important role in lung adenocarcinoma carcinogenesis, and may become diagnostic markers for lung cancer. However, this should be confirmed 
Table 2. Enriched functions for genes in upregulated modules with a higher enrichment score.

\begin{tabular}{|c|c|c|c|c|}
\hline Module/Term & Description & Count & $P$ & DEGs \\
\hline \multicolumn{5}{|l|}{ up-1 } \\
\hline GO:0000375 & $\begin{array}{l}\text { RNA splicing, via transesterification } \\
\text { reactions }\end{array}$ & 14 & $1.53 \mathrm{E}-17$ & $\begin{array}{c}\text { PABPN1, POLR2G, DHX9, POLR2F, HNRNPA2B1, } \\
\text { PTBP1, HNRNPR, DDX23, CLP1, GTF2F2... }\end{array}$ \\
\hline GO:0000377 & $\begin{array}{l}\text { RNA splicing, via transesterification } \\
\text { reactions with bulged adenosine as } \\
\text { nucleophile }\end{array}$ & 14 & $1.53 \mathrm{E}-17$ & $\begin{array}{c}\text { PABPN1, POLR2G, DHX9, POLR2F, HNRNPA2B1, } \\
\text { PTBP1, HNRNPR, DDX23, CLP1, GTF2F2... }\end{array}$ \\
\hline GO:0000398 & $\begin{array}{l}\text { Nuclear mRNA splicing, via } \\
\text { spliceosome }\end{array}$ & 14 & $1.53 \mathrm{E}-17$ & $\begin{array}{c}\text { PABPN1, POLR2G, DHX9, POLR2F, HNRNPA2B1, } \\
\text { PTBP1, HNRNPR, DDX23, CLP1, GTF2F2... }\end{array}$ \\
\hline GO:0008380 & RNA splicing & 14 & $1.03 \mathrm{E}-14$ & $\begin{array}{c}\text { PABPN1, POLR2G, DHX9, POLR2F, HNRNPA2B1, } \\
\text { PTBP1, HNRNPR, DDX23, CLP1, GTF2F2 .. }\end{array}$ \\
\hline GO:0006397 & mRNA processing & 14 & $3.94 \mathrm{E}-14$ & $\begin{array}{c}\text { PABPN1, POLR2G, DHX9, POLR2F, HNRNPA2B1, } \\
\text { PTBP1, HNRNPR, DDX23, CLP1, GTF2F2... }\end{array}$ \\
\hline GO:0016071 & mRNA metabolic process & 14 & $2.19 \mathrm{E}-13$ & $\begin{array}{c}\text { PABPN1, POLR2G, DHX9, POLR2F, HNRNPA2B1, } \\
\text { PTBP1, HNRNPR, DDX23, CLP1, GTF2F2... }\end{array}$ \\
\hline GO:0006396 & RNA processing & 14 & $7.55 \mathrm{E}-12$ & $\begin{array}{c}\text { PABPN1, POLR2G, DHX9, POLR2F, HNRNPA2B1, } \\
\text { PTBP1, HNRNPR, DDX23, CLP1, GTF2F2... }\end{array}$ \\
\hline \multicolumn{5}{|l|}{ up-2 } \\
\hline GO:0006119 & Oxidative phosphorylation & 12 & 3.69E-15 & $\begin{array}{l}\text { UQCRC2, ATP5D, NDUFS7, NDUFA2, UQCR11, } \\
\text { NDUFB8, NDUFV1, NDUFV2, NDUFA7, NDUFS3... }\end{array}$ \\
\hline GO:0042775 & $\begin{array}{l}\text { Mitochondrial ATP synthesis coupled } \\
\text { electron transport }\end{array}$ & 10 & $1.12 \mathrm{E}-14$ & $\begin{array}{l}\text { NDUFS7, NDUFA2, UQCR11, NDUFB8, NDUFV1, } \\
\text { NDUFV2, NDUFA7, NDUFS3, NDUFA1, NDUFB1 }\end{array}$ \\
\hline GO:0042773 & $\begin{array}{c}\text { ATP synthesis coupled electron } \\
\text { transport }\end{array}$ & 10 & $1.12 \mathrm{E}-14$ & $\begin{array}{l}\text { NDUFS7, NDUFA2, UQCR11, NDUFB8, NDUFV1, } \\
\text { NDUFV2, NDUFA7, NDUFS3, NDUFA1, NDUFB1 }\end{array}$ \\
\hline GO:0022904 & Respiratory electron transport chain & 10 & 7.35E-14 & $\begin{array}{l}\text { NDUFS7, NDUFA2, UQCR11, NDUFB8, NDUFV1, } \\
\text { NDUFV2, NDUFA7, NDUFS3, NDUFA1, NDUFB1 }\end{array}$ \\
\hline GO:0006120 & $\begin{array}{l}\text { Mitochondrial electron transport, } \\
\text { NADH to ubiquinone }\end{array}$ & 9 & $9.94 \mathrm{E}-14$ & $\begin{array}{c}\text { NDUFS7, NDUFA2, NDUFB8, NDUFV1, NDUFV2, } \\
\text { NDUFA7, NDUFS3, NDUFA1, NDUFB1 }\end{array}$ \\
\hline GO:0045333 & Cellular respiration & 11 & $2.95 \mathrm{E}-13$ & $\begin{array}{l}\text { UQCRC2, NDUFS7, NDUFA2, UQCR11, NDUFB8, } \\
\text { NDUFV1, NDUFV2, NDUFA7, NDUFS3, NDUFA1... }\end{array}$ \\
\hline GO:0022900 & Electron transport chain & 11 & $3.41 \mathrm{E}-13$ & $\begin{array}{l}\text { UQCRC2, NDUFS7, NDUFA2, UQCR11, NDUFB8, } \\
\text { NDUFV1, NDUFV2, NDUFA7, NDUFS3, NDUFA1... }\end{array}$ \\
\hline GO:0015980 & $\begin{array}{l}\text { Energy derivation by oxidation of } \\
\text { organic compounds }\end{array}$ & 11 & $2.93 \mathrm{E}-11$ & $\begin{array}{l}\text { UQCRC2, NDUFS7, NDUFA2, UQCR11, NDUFB8, } \\
\text { NDUFV1, NDUFV2, NDUFA7, NDUFS3, NDUFA1... }\end{array}$ \\
\hline GO:0006091 & $\begin{array}{c}\text { Generation of precursor metabolites } \\
\text { and energy }\end{array}$ & 13 & 1.87E-10 & $\begin{array}{l}\text { ATP5D, UQCRC2, NDUFA2, NDUFB8, NDUFA7, } \\
\text { COX4I1, NDUFA1, NDUFB1, NDUFS7, UQCR11... }\end{array}$ \\
\hline GO:0055114 & Oxidation reduction & 11 & $6.10 \mathrm{E}-06$ & $\begin{array}{l}\text { UQCRC2, NDUFS7, NDUFA2, UQCR11, NDUFB8, } \\
\text { NDUFV1, NDUFV2, NDUFA7, NDUFS3, NDUFA1... }\end{array}$ \\
\hline GO:0016310 & Phosphorylation & 12 & 3.35E-05 & $\begin{array}{l}\text { UQCRC2, ATP5D, NDUFS7, NDUFA2, UQCR11, } \\
\text { NDUFB8, NDUFV1, NDUFV2, NDUFA7, NDUFS3... }\end{array}$ \\
\hline GO:0006793 & Phosphorus metabolic process & 12 & $1.78 \mathrm{E}-04$ & $\begin{array}{l}\text { UQCRC2, ATP5D, NDUFS7, NDUFA2, UQCR11, } \\
\text { NDUFB8, NDUFV1, NDUFV2, NDUFA7, NDUFS3... }\end{array}$ \\
\hline GO:0006796 & Phosphate metabolic process & 12 & $1.78 \mathrm{E}-04$ & $\begin{array}{l}\text { UQCRC2, ATP5D, NDUFS7, NDUFA2, UQCR11, } \\
\text { NDUFB8, NDUFV1, NDUFV2, NDUFA7, NDUFS3... }\end{array}$ \\
\hline
\end{tabular}

GO: gene ontology; DEGs: differentially expressed genes.

in future in-depth studies. Similarly, UQCRC2 and UQCR11 may contribute to the occurrence of lung adenocarcinoma. They encode the ubiquinol-cytochrome $c$ reductase complex, which is responsible for carrying electrons from ubiquinol to cytochrome $c$ in the mitochondrial respiratory chain (31).
Moreover, ATP5D, which encodes a subunit of mitochondrial ATP synthase, is associated with mitochondrial ATP synthesis coupled electron transport in lung adenocarcinoma cells (32).

DEGs such as PRIM1, MCM3, and RNASEH2A, related to DNA replication, were also upregulated in lung 
Table 3. Enriched KEGG pathways for genes in upregulated modules with a higher enrichment score.

\begin{tabular}{ccccc}
\hline Module/Term & Description & Count & $P$ & DEGs \\
\hline $\begin{array}{c}\text { up-1 } \\
\text { hsa00240 }\end{array}$ & Pyrimidine metabolism & 4 & $1.15 \mathrm{E}-03$ & PRIM1, POLR2G, POLR2F, TK1 \\
hsa03030 & DNA replication & 3 & $5.24 \mathrm{E}-03$ & $P R I M 1, M C M 3, R N A S E H 2 A$ \\
hsa03040 & Spliceosome & 3 & $4.41 \mathrm{E}-02$ & DDX23, SNRNP40, SNRNP70 \\
up-2 & & & \\
hsa00190 & Oxidative phosphorylation & 13 & $3.31 \mathrm{E}-13$ & ATP5D, UQCRC2, NDUFA2, NDUFB8, NDUFA7, COX4I1, NDUFA1, \\
hsa05012 & Parkinson's disease & 13 & $3.81 \mathrm{E}-13$ & NDUFB1, NDUFS7, UQCR11, NDUFV1, NDUFV2, NDUFS3 \\
hsa05010 & Alzheimer's disease & 13 & $3.25 \mathrm{E}-11$ & \\
hsa05016 & Huntington's disease & 13 & $7.35 \mathrm{E}-11$ & \\
hsa03050 & Proteasome & 7 & $3.50 \mathrm{E}-07$ & PSMB10, PSMC6, PSMD13, PSMB6, PSMC5, PSME1, PSMC3 \\
hsa03010 & Ribosome & 6 & $2.09 \mathrm{E}-04$ & RPL30, RPLP1, RPL27, FAU, RPL37, RPL38 \\
\hline
\end{tabular}

KEGG: Kyoto Encyclopedia of Genes and Genomes; DEGs: differentially expressed genes.

Table 4. Enriched functions for genes in downregulated modules with a higher enrichment score.

\begin{tabular}{|c|c|c|c|c|}
\hline Module/Term & Description & Count & $\mathrm{P}$ & DEGs \\
\hline \multicolumn{5}{|c|}{$\mathrm{d}-1$ (Enrichment score $=2.57)$} \\
\hline GO:0007166 & $\begin{array}{l}\text { Cell surface receptor linked signal } \\
\text { transduction }\end{array}$ & 8 & $1.71 \mathrm{E}-03$ & $\begin{array}{c}\text { MAPK1, MAPK14, MITF, RAF1, JAK1, HBEGF, } \\
\text { ROS1, STAT3 }\end{array}$ \\
\hline GO:0007167 & $\begin{array}{l}\text { Enzyme linked receptor protein } \\
\text { signaling pathway }\end{array}$ & 5 & 2.02E-03 & RAF1, JAK1, HBEGF, ROS1, STAT3 \\
\hline GO:0007169 & $\begin{array}{l}\text { Transmembrane receptor protein } \\
\text { tyrosine kinase signaling pathway }\end{array}$ & 4 & 5.64E-03 & RAF1, HBEGF, ROS1, STAT3 \\
\hline \multicolumn{5}{|c|}{$\mathrm{d}-1$ (Enrichment score $=2.05)$} \\
\hline GO:0007166 & $\begin{array}{l}\text { Cell surface receptor linked signal } \\
\text { transduction }\end{array}$ & 8 & $1.71 \mathrm{E}-03$ & $\begin{array}{c}\text { MAPK1, MAPK14, MITF, RAF1, JAK1, HBEGF, } \\
\text { ROS1, STAT3 }\end{array}$ \\
\hline GO:0007242 & Intracellular signaling cascade & 7 & 4.96E-03 & ZFP36, MAPK1, DUSP1, MAPK14, RAF1, JAK1, STAT3 \\
\hline GO:0006793 & Phosphorus metabolic process & 6 & 9.78E-03 & MAPK1, DUSP1, MAPK14, RAF1, JAK1, ROS1 \\
\hline GO:0006796 & Phosphate metabolic process & 6 & $9.78 \mathrm{E}-03$ & MAPK1, DUSP1, MAPK14, RAF1, JAK1, ROS1 \\
\hline GO:0006468 & Protein amino acid phosphorylation & 5 & 1.42E-02 & MAPK1, MAPK14, RAF1, JAK1, ROS1 \\
\hline GO:0007265 & Ras protein signal transduction & 3 & 1.44E-02 & MAPK1, MAPK14, RAF1 \\
\hline GO:0016310 & Phosphorylation & 5 & 2.54E-02 & MAPK1, MAPK14, RAF1, JAK1, ROS1 \\
\hline \multicolumn{5}{|c|}{ d-5 (Enrichment score $=2.22)$} \\
\hline GO:0046488 & $\begin{array}{l}\text { Phosphatidylinositol metabolic } \\
\text { process }\end{array}$ & 3 & 4.24E-04 & PIP5K1B, PIP5K1C, PIP4K2B \\
\hline GO:0046486 & Glycerolipid metabolic process & 4 & 1.88E-03 & CAV1, PIP5K1B, PIP5K1C, PIP4K2B \\
\hline GO:0030384 & Phosphoinositide metabolic process & 3 & 5.23E-03 & PIP5K1B, PIP5K1C, PIP4K2B \\
\hline GO:0006650 & Glycerophospholipid metabolic process & 3 & 1.38E-02 & PIP5K1B, PIP5K1C, PIP4K2B \\
\hline GO:0006644 & Phospholipid metabolic process & 3 & 2.76E-02 & PIP5K1B, PIP5K1C, PIP4K2B \\
\hline GO:0019637 & Organophosphate metabolic process & 3 & $3.12 \mathrm{E}-02$ & PIP5K1B, PIP5K1C, PIP4K2B \\
\hline \multicolumn{5}{|c|}{ d-5 $($ Enrichment score $=1.83)$} \\
\hline GO:0032989 & Cellular component morphogenesis & 5 & 3.71E-03 & MYH11, GJA1, PIP5K1C, CXCL12, FN1 \\
\hline GO:0031175 & Neuron projection development & 4 & 9.35E-03 & IL6, GJA1, PIP5K1C, CXCL12 \\
\hline GO:0048666 & Neuron development & 4 & 1.90E-02 & IL6, GJA1, PIP5K1C, CXCL12 \\
\hline GO:0000902 & Cell morphogenesis & 4 & 2.06E-02 & GJA1, PIP5K1C, CXCL12, FN1 \\
\hline GO:0030030 & Cell projection organization & 4 & 2.16E-02 & IL6, GJA1, PIP5K1C, CXCL12 \\
\hline GO:0030182 & Neuron differentiation & 4 & 3.39E-02 & IL6, GJA1, PIP5K1C, CXCL12 \\
\hline
\end{tabular}

GO: gene ontology; DEGs: differentially expressed genes. 
Table 5. Enriched KEGG pathways for genes in downregulated modules with a higher enrichment score.

\begin{tabular}{|c|c|c|c|c|}
\hline Module/Term & Description & Count & $\mathrm{P}$ & DEGs \\
\hline \multicolumn{5}{|c|}{$\mathrm{d}-1$ (Enrichment score $=2.60)$} \\
\hline hsa05212 & Pancreatic cancer & 4 & 5.47E-04 & MAPK1, RAF1, JAK1, STAT3 \\
\hline hsa05200 & Pathways in cancer & 5 & 3.69E-03 & $\begin{array}{c}\text { MAPK1, MITF, RAF1, JAK1, } \\
\text { STAT3 }\end{array}$ \\
\hline hsa05221 & Acute myeloid leukemia & 3 & 7.67E-03 & MAPK1, RAF1, STAT3 \\
\hline \multicolumn{5}{|c|}{$\mathrm{d}-1($ Enrichment score $=2.11)$} \\
\hline hsa05200 & Pathways in cancer & 5 & 3.69E-03 & $\begin{array}{c}\text { MAPK1, MITF, RAF1, JAK1, } \\
\text { STAT3 }\end{array}$ \\
\hline hsa05218 & Melanoma & 3 & $8.52 \mathrm{E}-03$ & MAPK1, MITF, RAF1 \\
\hline hsa04916 & Melanogenesis & 3 & $1.48 \mathrm{E}-02$ & MAPK1, MITF, RAF1 \\
\hline \multicolumn{5}{|c|}{ d-5 $($ Enrichment score $=1.76)$} \\
\hline hsa04810 & Regulation of actin cytoskeleton & 5 & 4.61E-03 & $\begin{array}{c}\text { PIP5K1B, PIP5K1C, FN1, } \\
\text { PIP4K2B, MYL9 }\end{array}$ \\
\hline hsa00562 & Inositol phosphate metabolism & 3 & $1.56 \mathrm{E}-02$ & PIP5K1B, PIP5K1C, PIP4K2B \\
\hline hsa04070 & Phosphatidylinositol signaling system & 3 & $2.90 \mathrm{E}-02$ & PIP5K1B, PIP5K1C, PIP4K2B \\
\hline hsa04666 & Fc gamma R-mediated phagocytosis & 3 & 4.57E-02 & PIP5K1B, PIP5K1C, PIP4K2B \\
\hline
\end{tabular}

KEGG: Kyoto Encyclopedia of Genes and Genomes; DEGs: differentially expressed genes.

adenocarcinoma cells in the present study. Lung adenocarcinoma is a form of solid tumor, and its biological behavior including formation, development, and attack is closely related to abnormal cell proliferation, which includes DNA replication. PRIM1 encodes one of the subunits ( $p 49$ ) of the eukaryotic primase, which is a heterodimer consisting of a small and a large subunit that synthesizes RNA primers for the Okazaki fragments during discontinuous DNA replication (33). MCM3 encodes a major control factor in eukaryotic DNA replication initiation and extension $(34,35)$, while RNASEH2A codes for a subunit of the ribonuclease $\mathrm{H} 2$ complex which helps break down RNA from RNA-DNA hybrids formed during DNA replication (36). Therefore, these identified DEGs are thought to play important roles in lung adenocarcinoma.

Some DEGs in downregulated modules, such as MAPK1, STAT3, RAF1, and JAK1, are enriched in cell surface receptor linked signal transduction and the enzyme linked receptor protein signaling pathway. MAPK1 in the MAPK family is also known as extracellular signal-regulated kinase2 (ERK2), and is involved in cell proliferation. Moreover, the ERK pathway is known to be activated during the early stages of lung adenocarcinoma $(37,38)$. RAF is a proto-oncogene, encoding a serine/threonine protein kinase that functions in the highly conserved Ras-Raf-MEK-ERK signal transduction pathway, and provides an important link between Ras and ERK signaling activation. RAF1 in the RAF family encodes Raf1 kinase, which is reported to be expressed abnormally in human lung adenocarcinomas (39). Finally, the JAK/STAT3 signaling pathway plays an essential part in the formation of NSCLC (40). Hence, it appears that these genes might be involved in the formation and development of lung adenocarcinoma.

In conclusion, we have identified DEGs that might be involved in the pathogenesis of lung adenocarcinoma. In particular, the upregulated DEGs (e.g., PRIM1, MCM3, RNASEH2A, UQCRC2, UQCR11I, ATP5D, PRIM1, MCM3, RNASEH2A, and DEGs encoding subunits of NADH) and downregulated DEGs (e.g., MAPK1, STAT3, RAF1, and $J A K 1$ ) in network modules related to important functions and pathways might provide novel insights into the molecular mechanisms underlying lung adenocarcinoma and serve as therapeutic targets. However, our findings should be confirmed by further experiments, and lung adenocarcinoma at different stages should be investigated.

\section{Supplementary Material}

Click here to view [pdf]

\section{Acknowledgements}

We thank Harbin Medical University College of Bioinformatics Science and Technology for help with the analysis of biological information data. This study was funded by the Health and Family Planning Commission of Heilongjiang Province (Grant \#2014-359). 


\section{References}

1. Chen WQ, Zhang SW, Zou XN. Estimation and projection of lung cancer incidence and mortality in China. Chin J Lung Cancer 2010; 13: 488-493.

2. Parkin DM, Bray F, Ferlay J, Pisani P. Global cancer statistics, 2002. CA Cancer J Clin 2005; 55: 74-108, doi: 10.3322/canjclin. 55.2.74.

3. Kris $M$, Johnson $B$, Kwiatkowski D, lafrate A, Wistuba I, Aronson $\mathrm{S}$, et al. Identification of driver mutations in tumor specimens from 1,000 patients with lung adenocarcinoma: The NCl's Lung Cancer Mutation Consortium (LCMC). $J$ Clin Oncol 2011; 29 (15 Suppl).

4. Mollberg N, Surati M, Demchuk C, Fathi R, Salama AK, Husain AN, et al. Mind-mapping for lung cancer: towards a personalized therapeutics approach. Adv Ther 2011; 28: 173-194, doi: 10.1007/s12325-010-0103-9.

5. Van Damme N, Deron P, Van Roy N, Demetter P, Bols A, Van Dorpe J, et al. Epidermal growth factor receptor and K-RAS status in two cohorts of squamous cell carcinomas. BMC Cancer 2010; 10: 189, doi: 10.1186/1471-2407-10-189.

6. Forbes S, Clements J, Dawson E, Bamford S, Webb T, Dogan A, et al. COSMIC 2005. Br J Cancer 2006; 94: 318-322, doi: 10.1038/sj.bjc.6602928.

7. Mills NE, Fishman CL, Scholes J, Anderson SE, Rom WN, Jacobson DR. Detection of K-ras oncogene mutations in bronchoalveolar lavage fluid for lung cancer diagnosis. J Natl Cancer Inst 1995; 87: 1056-1060, doi: 10.1093/jnci/87.14.1056.

8. Zhang X, Zhang S, Yang X, Yang J, Zhou Q, Yin L, et al. Fusion of EML4 and ALK is associated with development of lung adenocarcinomas lacking EGFR and KRAS mutations and is correlated with ALK expression. Mol Cancer 2010; 9: 188, doi: 10.1186/1476-4598-9-188.

9. Chapman PB, Hauschild A, Robert C, Haanen JB, Ascierto $\mathrm{P}$, Larkin $\mathrm{J}$, et al. Improved survival with vemurafenib in melanoma with BRAF V600E mutation. N Engl J Med 2011; 364: 2507-2516, doi: 10.1056/NEJMoa1103782.

10. Takeuchi K, Soda M, Togashi Y, Suzuki R, Sakata S, Hatano $\mathrm{S}$, et al. RET, ROS1 and ALK fusions in lung cancer. Nat Med 2012; 18: 378-381, doi: 10.1038/nm.2658.

11. Jun $H J$, Johnson $H$, Bronson $R T$, de Feraudy $S$, White $F$, Charest $A$. The oncogenic lung cancer fusion kinase CD74-ROS activates a novel invasiveness pathway through E-Syt1 phosphorylation. Cancer Res 2012; 72: 3764-3774, doi: 10.1158/0008-5472.CAN-11-3990.

12. Friboulet L, Olaussen KA, Pignon JP, Shepherd FA, Tsao MS, Graziano S, et al. ERCC1 isoform expression and DNA repair in non-small-cell lung cancer. N Engl J Med 2013; 368: 1101-1110, doi: 10.1056/NEJMoa1214271.

13. Kohno S, Kitajima S, Sasaki N, Muranaka H, Takahashi C. Abstract LB-130: The metabolic function of RB tumor suppressor gene. Cancer Research 2013; 73 (8 Supplement): LB-130, doi: 10.1158/1538-7445.AM2013-LB-130.

14. Heavey S, O'Byrne KJ, Gately K. Strategies for co-targeting the PI3K/AKT/mTOR pathway in NSCLC. Cancer Treat Rev 2014; 40: 445-456, doi: 10.1016/j.ctrv.2013.08.006.

15. Minami D, Takigawa N, Takeda H, Takata M, Ochi N, Ichihara E, et al. PTEN deficient lung cancer cells are sensitive to the combination of olaparib with cisplatin through suppressing DNA damage signaling. Cancer Research 2013; 73 (8 Supplement): 2048, doi: 10.1158/1538-7445.AM2013-2048.
16. Marks JL, Gong Y, Chitale D, Golas B, McLellan MD, Kasai Y, et al. Novel MEK1 mutation identified by mutational analysis of epidermal growth factor receptor signaling pathway genes in lung adenocarcinoma. Cancer Res 2008; 68: 5524-5528, doi: 10.1158/0008-5472.CAN-08-0099.

17. Stearman RS, Dwyer-Nield L, Zerbe L, Blaine SA, Chan Z, Bunn PA Jr, et al. Analysis of orthologous gene expression between human pulmonary adenocarcinoma and a carcinogeninduced murine model. Am J Pathol 2005; 167: 1763-1775, doi: 10.1016/S0002-9440(10)61257-6.

18. Wu Z, Irizarry R A, Gentleman R, Martinez-Murillo F, Spencer F. A model-based background adjustment for oligonucleotide expression arrays. J Amer Statist Assoc 2004; 99: 909-917, doi: 10.1198/016214504000000683.

19. Benjamini $Y$, Hochberg $Y$. Controlling the false discovery rate: a practical and powerful approach to multiple testing. J R Stat Soc Ser B Stat Methodol 1995; 289-300.

20. Kanehisa M, Goto S. KEGG: kyoto encyclopedia of genes and genomes. Nucleic Acids Res 2000; 28: 27-30, doi: 10.1093/ nar/28.1.27.

21. Lamb J, Crawford ED, Peck D, Modell JW, Blat IC, Wrobel MJ, et al. The Connectivity Map: using gene-expression signatures to connect small molecules, genes, and disease. Science 2006; 313: 1929-1935, doi: 10.1126/science.1132939.

22. He X, Zhang J. Why do hubs tend to be essential in protein networks? PLoS Genet 2006; 2: e88, doi: 10.1371/journal. pgen.0020088.

23. Emahazion T, Brookes AJ. Mapping of the NDUFA2, NDUFA6, NDUFA7, NDUFB8, and NDUFS8 electron transport chain genes by intron based radiation hybrid mapping. Cytogenet Cell Genet 1998; 82: 114, doi: 10.1159/000015081.

24. Nakamaru-Ogiso E, Han H, Matsuno-Yagi A, Keinan E, Sinha SC, Yagi T, et al. The ND2 subunit is labeled by a photoaffinity analogue of asimicin, a potent complex I inhibitor. FEBS Lett 2010; 584: 883-888, doi: 10.1016/j.febslet.2010. 01.004.

25. Guppy M, Leedman P, Zu X, Russell V. Contribution by different fuels and metabolic pathways to the total ATP turnover of proliferating MCF-7 breast cancer cells. Biochem J 2002; 364: 309-315, doi: 10.1042/bj3640309.

26. Griguer CE, Oliva CR, Gillespie GY. Glucose metabolism heterogeneity in human and mouse malignant glioma cell lines. J Neurooncol 2005; 74: 123-133, doi: 10.1007/s11060004-6404-6.

27. Herst PM, Berridge MV. Cell surface oxygen consumption: a major contributor to cellular oxygen consumption in glycolytic cancer cell lines. Biochim Biophys Acta 2007; 1767: 170-177, doi: 10.1016/j.bbabio.2006.11.018.

28. Hooda J, Cadinu D, Alam MM, Shah A, Cao TM, Sullivan LA, et al. Enhanced heme function and mitochondrial respiration promote the progression of lung cancer cells. PLOS One 2013; 8: e63402, doi: 10.1371/journal.pone.0063402.

29. Han YH, Kim SH, Kim SZ, Park WH. Antimycin A as a mitochondrial electron transport inhibitor prevents the growth of human lung cancer A549 cells. Oncol Rep 2008; 20: 689-693.

30. Su CY, Hsiao M. NDUFS1 as a prognostic marker in lung cancer: a clinicopathological analysis of 106 cases of nonsmall cell lung cancer. FASEB J 2013; 27: 58.57. 
31. Miyake N, Yano S, Sakai C, Hatakeyama H, Matsushima Y, Shiina M, et al. Mitochondrial complex III deficiency caused by a homozygous UQCRC2 mutation presenting with neonatalonset recurrent metabolic decompensation. Hum Mutat 2013; 34: 446-452, doi: 10.1002/humu.22257.

32. Chen G, Gharib TG, Huang CC, Thomas DG, Shedden KA, Taylor JM, et al. Proteomic analysis of lung adenocarcinoma: identification of a highly expressed set of proteins in tumors. Clin Cancer Res 2002; 8: 2298-2305.

33. Copeland WC, Tan X. Active site mapping of the catalytic mouse primase subunit by alanine scanning mutagenesis. J Biol Chem 1995; 270: 3905-3913, doi: 10.1074/jbc.270.8.3905.

34. Bell SP, Dutta A. DNA replication in eukaryotic cells. Annu Rev Biochem 2002; 71: 333-374, doi: 10.1146/annurev. biochem.71.110601.135425.

35. Schwacha A, Bell SP. Interactions between two catalytically distinct MCM subgroups are essential for coordinated ATP hydrolysis and DNA replication. Mol Cell 2001; 8: 1093-1104, doi: 10.1016/S1097-2765(01)00389-6.

36. Crow YJ, Leitch A, Hayward BE, Garner A, Parmar R, Griffith $E$, et al. Mutations in genes encoding ribonuclease $\mathrm{H} 2$ subunits cause Aicardi-Goutieres syndrome and mimic congenital viral brain infection. Nat Genet 2006; 38: 910-916, doi: 10.1038/ng1842.

37. Voisin L, Saba-El-Leil MK, Julien C, Fremin C, Meloche S. Genetic demonstration of a redundant role of extracellular signal-regulated kinase 1 (ERK1) and ERK2 mitogenactivated protein kinases in promoting fibroblast proliferation. Mol Cell Biol 2010; 30: 2918-2932, doi: 10.1128/ MCB.00131-10.

38. Lopez-Malpartida AV, Ludena MD, Varela G, Garcia PJ. Differential ErbB receptor expression and intracellular signaling activity in lung adenocarcinomas and squamous cell carcinomas. Lung Cancer 2009; 65: 25-33, doi: 10.1016/ j.lungcan.2008.10.009.

39. Powell CA, Spira A, Derti A, DeLisi C, Liu G, Borczuk A, et al. Gene expression in lung adenocarcinomas of smokers and nonsmokers. Am J Respir Cell Mol Biol 2003; 29:157-162, doi: $10.1165 / \mathrm{rcmb} .2002-0183 R C$

40. Song L, Rawal B, Nemeth JA, Haura EB. JAK1 activates STAT3 activity in non-small-cell lung cancer cells and IL-6 neutralizing antibodies can suppress JAK1-STAT3 signaling. Mol Cancer Ther 2011; 10: 481-494, doi: 10.1158/15357163.MCT-10-0502. 